\title{
Electrokinetic Analysis of Poorly Conductive
}

\section{Electrocatalytic Materials}

Dong Young Chung, ${ }^{\dagger+}$ Subin Park,,$\|\|+$, Pietro P. Lopes, ${ }^{\dagger}$ Vojislav R. Stamenkovic,,$^{\dagger}$ Yung-Eun Sung, $\#, \| *$ Nenad M. Markovic, ${ }^{\dagger *}$ Dusan Strmcnik ${ }^{\dagger *}$

${ }^{\dagger}$ Materials Science Division, Argonne National Laboratory, Lemont, IL 60439, United States

${ }^{\#}$ Center for Nanoparticle Research, Institute for Basic Science (IBS), Seoul 08826, Republic of Korea

${ }$ School of Chemical and Biological Engineering, Seoul National University (SNU), Seoul 08826, Republic of Korea

\section{Corresponding Author \\ *strmcnik@anl.gov \\ *ysung@snu.ac.kr \\ *nmmarkovic@anl.gov}




\section{Experimental section}

\section{Preparation of $\mathrm{MoS}_{2}$ samples.}

Commercially available reagents were used without further purification. Ammonium tetrathiomolybdate $\left(\left(\mathrm{NH}_{4}\right)_{2} \mathrm{MoS}_{4}\right.$, Sigma Aldrich) were grinded into fine powder using agate mortar. The grinded powder was then annealed at target temperature for amorphous $\mathrm{MoS}_{\mathrm{x}}$ $\left(250^{\circ} \mathrm{C}\right)$ and crystalline $\mathrm{MoS}_{2}\left(700^{\circ} \mathrm{C}\right)$ for $1 \mathrm{hr}$ in $\mathrm{Ar}$ atmosphere. The ramping rate is $5{ }^{\circ} \mathrm{C}$ $\min ^{-1}$.

\section{Physical characterization}

XRD patterns were obtained using a Rigaku (D-MAX 2500-PC) with $\mathrm{Cu}$ K $\alpha$ radiation source $(\lambda=0.15418 \mathrm{~nm}$ ) using $40 \mathrm{kV}$ and $200 \mathrm{~mA}$. TEM images were obtained by F20 (FEG Tecnai with $200 \mathrm{kV}$, equipped in Research Institute of Advanced Materials (RIAM) at Seoul National University). X-ray absorption fine structure (XAFS) analysis was conducted by Nanoprobe XAFS beamline (8C, Pohang Accelerator Laboratory (PAL), Pohang, Republic of Korea). All spectra were measured by transmission mode and energy was calibrated by reference Mo film before and during measurements. The data treatment was conducted using ATHENA software (Ifeffit packaging).

\section{Electronic resistance of electrode measurement}

The electronic resistances of electrode were obtained using four-point probe device (CMT-SP $2000 \mathrm{~N}$ ). The as prepared $\mathrm{MoS}_{2}$ powder was mixed with certain amount of carbon (Vulcan $\mathrm{XC}-72 \mathrm{R}$ ) in target ratio (see table S1) with Nafion ionomer (Nafion perfluorinated resin, 5 wt. $\%$ in mixture of lower aliphatic alcohols and water, Sigma Aldrich) as a binder. The composite ratio is identical with electrode preparation for electrochemical analysis (Table S1). The mixture was then pelletized into pellets $(10 \mathrm{~mm}$ diameter, $1 \mathrm{~mm}$ thickness $)$. The resistance of the pellet was measured at least 40 times at different positions. The electrode ohmic resistance was derived from resistivity, measured by four point-probe method, and known geometry of the electrode composite for electrochemical measurement.

\section{Electrochemical measurement}

Electrochemical measurements were controlled using an Autolab PGSTAT 302N potentiostat. A typical three-electrode configuration with fluoropolymer based cell was used. The electrode was prepared on glassy carbon electrode $\left(6 \mathrm{~mm}\right.$ diameter, $\left.0.283 \mathrm{~cm}^{-2}\right)$ with ink mixture of $\mathrm{MoS}_{2}$, carbon (Vulcan XC-72R) and Nafion as binder. The mass loading of $\mathrm{MoS}_{2}$ was fixed as $0.25 \mathrm{mg} \mathrm{cm}^{-2}$ and carbon content was controlled (see Table S1). Glassy carbon rod and $\mathrm{Ag} / \mathrm{AgCl}$ were used as counter and reference electrode, respectively. The $i R$ drop compensation was conducted during measurement $(5 \Omega)$. The current density in our paper was normalized by the geometric area $\left(0.283 \mathrm{~cm}^{2}\right)$. The EIS was measured under a constant voltage ( $0 \mathrm{~V}$ vs. RHE) in Ar saturated condition, using FRA32 (Autolab). The frequency range is from $100 \mathrm{mHz}$ to $100 \mathrm{kHz}$ with a $10 \mathrm{mV}$ amplitude. 

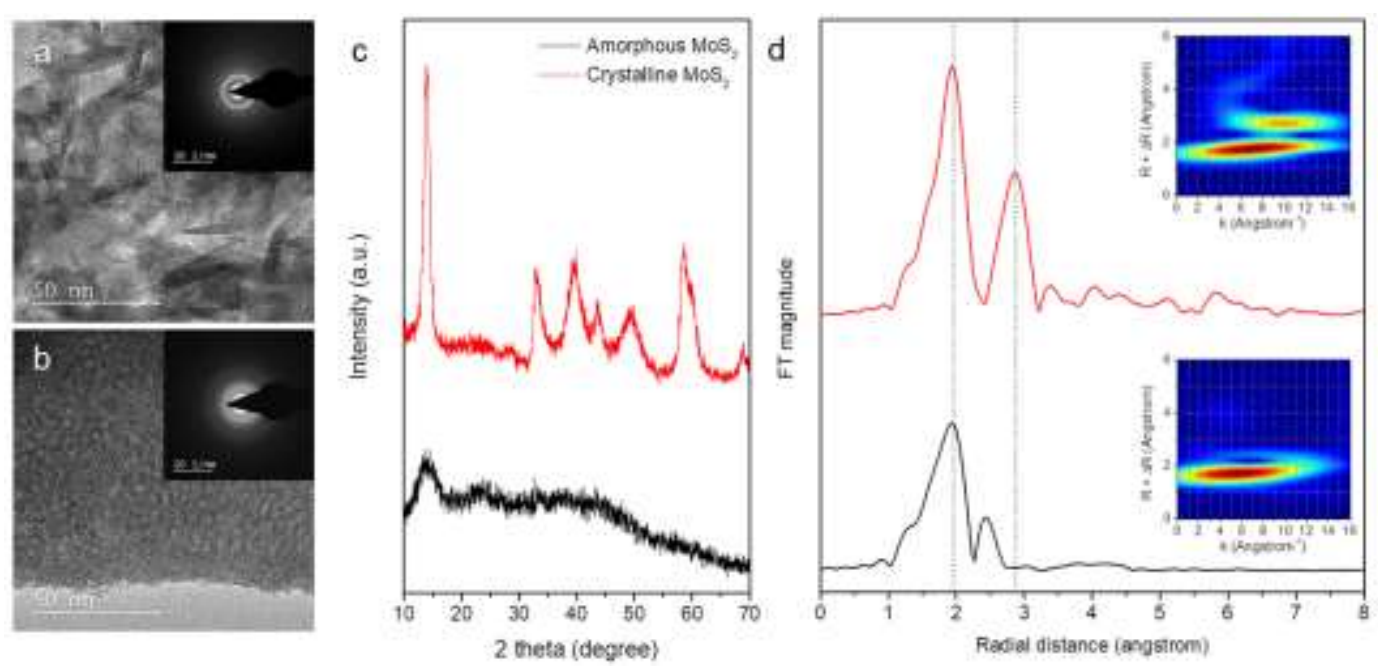

Figure S1. TEM images of (a) crystalline $\mathrm{MoS}_{2}$ and (b) amorphous $\mathrm{MoS}_{2}$ (Inset shows fast Fourier Transform results of TEM image) (c) XRD results of $\mathrm{MoS}_{2}$ samples and (d) Mo Kedge EXAFS results red (top): crystalline $\mathrm{MoS}_{2}$ and black (bottom): amorphous $\mathrm{MoS}_{2}$ (Inset shows wavelet transform results)

\section{Note 1}

\section{Characterization of crystalline $\mathrm{MoS}_{2}$ and amorphous $\mathrm{MoS}_{\mathbf{x}}$}

Highly crystalline and amorphous $\mathrm{MoS}_{2}$ was selected because significant activity difference has been reported while exact origin is still debate. To compare electrode ohmic resistance effect by adding carbon as conducting agent, carbon free $\mathrm{MoS}_{2}$ was synthesized. As shown in Figure S1a, typical 2 dimensional features of $\mathrm{MoS}_{2}$ were observed with stacking layer in crystalline $\mathrm{MoS}_{2}$ while stacking layer was not observed in amorphous one (Figure S1b). Fast Fourier transform analysis (inset of Figure S1a and S1b) and XRD (Figure S1c) supports that clear $2 \mathrm{H} \mathrm{MoS} 2$ features of crystalline $\mathrm{MoS}_{2}$ was observed. To clearly compare sample property, extended $\mathrm{x}$-ray absorption fine structure (EXAFS) analysis was conducted using Mo K-edge (Figure S1d). In Figure S1d, crystalline $\mathrm{MoS}_{2}$ shows typical $2 \mathrm{H} \mathrm{MoS} 2$ structure with Mo-S (around $2 \AA$ without phase shift correction) and Mo-Mo coordination (around $2.7 \AA$ ) while amorphous $\mathrm{MoS}_{2}$ shows only one feature in first coordination which is typical feature of amorphous. Wavelet transform image of two samples (inset of Figure S1d) also clearly support features of highly crystalline and amorphous feature, respectively. 
Table S1. Sample loading information; the identical amount of $\mathrm{MoS}_{2}$ loading was used in all cases (The geometric surface area of glassy carbon electrode is $0.283 \mathrm{~cm}^{2}$ ).

\begin{tabular}{|c|c|c|c|}
\hline Sample & $\begin{array}{l}\text { Mass loading of } \\
\mathrm{MoS}_{2}\left(\mathrm{mg} \mathrm{cm}^{-2}\right)\end{array}$ & $\begin{array}{l}\text { Mass loading of } \\
\text { carbon }\left(\mathrm{mg} \mathrm{cm}^{-2}\right)\end{array}$ & $\begin{array}{c}\text { Mass ratio } \\
\left(\mathrm{MoS}_{2}: \text { Carbon) }\right.\end{array}$ \\
\hline Without carbon & 0.25 & 0 & $1: 0$ \\
\hline 0.0125 & 0.25 & 0.0003125 & 1: 0.0125 \\
\hline 0.025 & 0.25 & 0.00625 & 1: 0.025 \\
\hline 0.05 & 0.25 & 0.0125 & 1: 0.05 \\
\hline 0.1 & 0.25 & 0.025 & 1: 0.1 \\
\hline 0.2 & 0.25 & 0.05 & 1: 0.2 \\
\hline 0.3 & 0.25 & 0.075 & 1: 0.3 \\
\hline 0.4 & 0.25 & 0.1 & 1: 0.4 \\
\hline
\end{tabular}


a

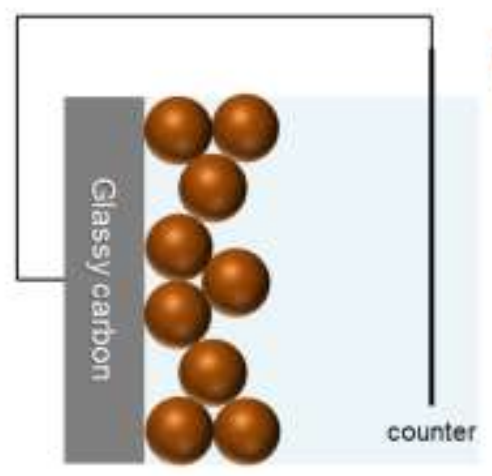

b

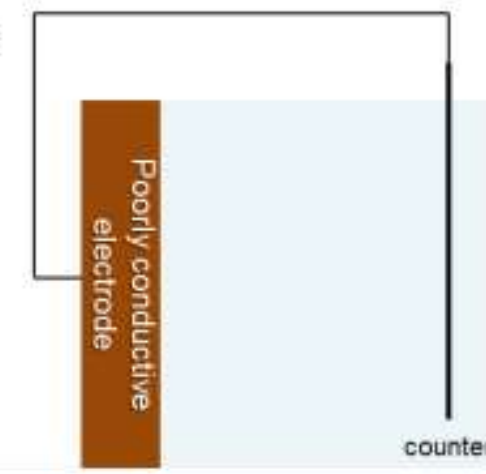

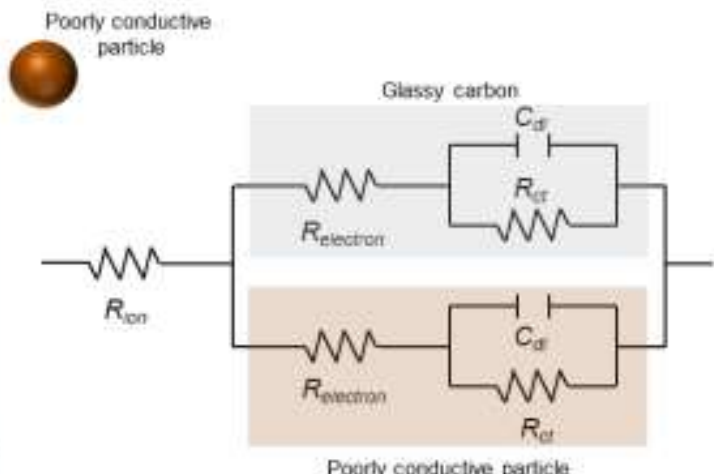

Poorly condictive partick



Poorly conductive electrodo

Figure S2. Scheme of electrochemical interface and that of electrochemical impedance spectroscopy circuit of (a) porous electrode and (b) film using poorly conductive materials as active materials.

\section{Note 2.}

\section{Electrochemical Impedance Spectroscopy (EIS) interpretation of iR correction}

The series resistance between the working electrode with the deposited composite material and the counter electrode, as measured by electrochemical impedance spectroscopy (EIS) at very high frequency (Figure S2), was unaffected by the $\mathrm{MoS}_{2}$ :carbon ratio and was stable at around $5 \Omega$, which is typical solution resistance $\left(\mathrm{R}_{\text {ion }}\right)$ value at $0.5 \mathrm{M} \mathrm{H}_{2} \mathrm{SO}_{4}$ (room temperature). Because a capacitor behaves as metallic conductor at high frequencies, on a well-porous composite electrode (Figure S2a), the path of least resistance will be given by the combination of solution resistance (ion transport) and the glassy carbon electronic conductivity, which is order of magnitude less resistive than $\mathrm{MoS}_{2}$ composites. Thus, at high frequency only solution resistance can be shown in impedance, which was compensated in all of our polarization (i-E) curve measurements. If there is no direct contact between backing electrode (in our case; glassy carbon) and electrolyte (Figure S2b), the ohmic resistance (Relectron_active materials) of the active materials would contribute to the overall pathway, and the

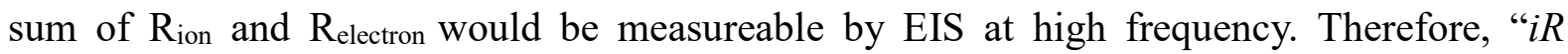
compensation" (during or after measurement) using EIS in porous electrode does not include 
compensation of "electrode ohmic resistance". It is worth mentioning that under catalytic conditions, which can be thought of being equivalent to low frequency in EIS, the path of least resistance in Figure S2a inverts, as $\mathrm{R}_{\mathrm{ct}}$ for the $\mathrm{MoS}_{2}$ composite is much smaller than the $\mathrm{R}_{\mathrm{ct}}$ for glassy carbon surface (poor catalyst for HER). Thus, the current will be influenced by the poor electronic conduction of $\mathrm{MoS}_{2}$ particles, giving rise to the deviations in the polarization curves as a function of the composite conductivity. 



Figure S3. Comparison of electrode ohmic resistance and HER overpotential (to reach $10 \mathrm{~mA} \mathrm{~cm}^{-2}$ ) (a) crystalline $\mathrm{MoS}_{2}$ and (b) amorphous $\mathrm{MoS}_{2}(\mathrm{MoSx})$. Blue shade region indicates where electrical percolation network is formed in electrode. The identical trend of activity and electrode ohmic resistance indicates that active $\mathrm{MoS}_{2}$ materials are well dispersed in carbon network. 

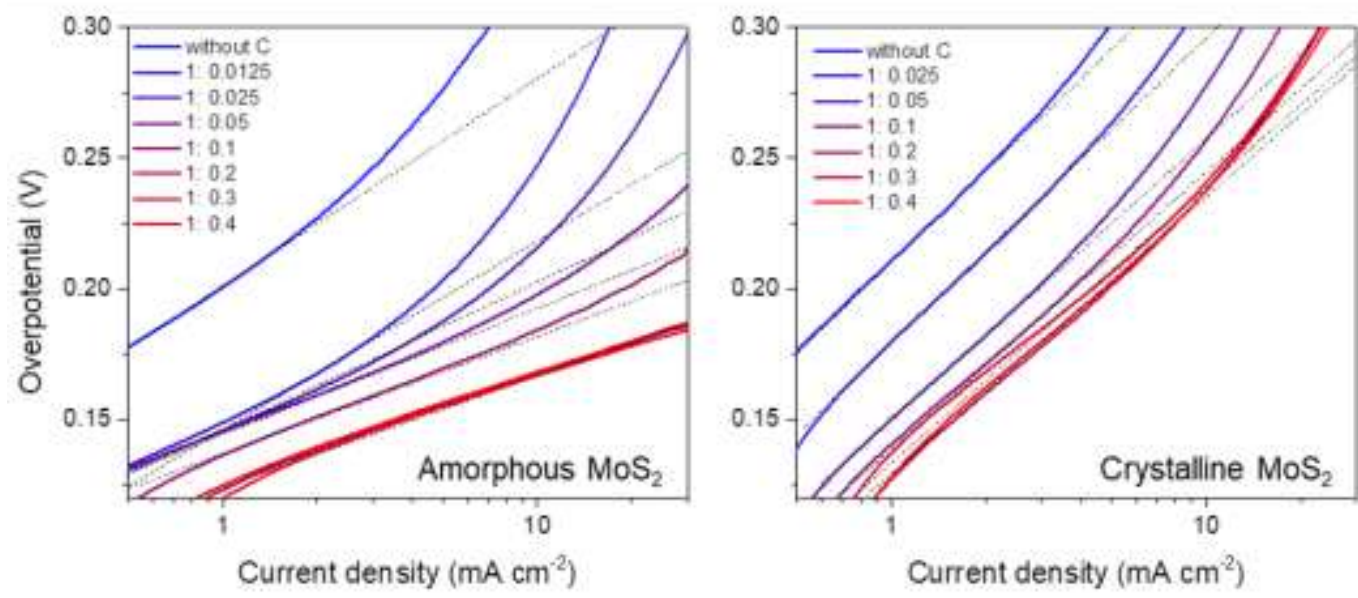

Figure S4. Log [current] versus overpotential plot of amorphous and crystalline $\mathrm{MoS}_{2}$. The Tafel slope (dash) was obtained at linear region $\left(2 \mathrm{~mA} \mathrm{~cm}^{-2}\right)$

\section{Note 3.}

\section{Utilization of electrode $(\theta)$ and Tafel slope correction using measured electrode ohmic resistance}

The utilization of electrode $(\theta)$ can be calculated based on the equation 3 in main text $=$ $\frac{i_{0}}{i_{0} \text { (ideal) }}=\frac{A}{A \text { (ideal) }}$. From the Figure S4, we obtain $\mathrm{i}_{0}$ in every carbon composition and calculate $\theta$ using io (ideal) where carbon ratio is 0.4 (Figure 3a in main text). From the equation 6 in main text $\left(\mathrm{b}=\frac{d \eta}{d \ln i}-\frac{R i}{3}\right)$, we can get "electrical ohmic loss corrected Tafel slope (b)" from the "observed Tafel slope $\left(\frac{d \eta}{d \ln i}\right)$ ", which is obtained by tangent line from Figure S4 and electrode composite resistance values (R), as determined in Figure 1a and $1 \mathrm{~b}$ in the main text (the whole process was done at current density of $2 \mathrm{~mA} \mathrm{~cm}^{-2}$ ). From the equation 6, the observed Tafel slope is closer to the "electrical ohmic loss corrected Tafel slope" as the ohmic resistance decreases, showing that $\frac{R i}{3}$ is the deviation value originated by the electrical conductivity issues. As discussed in manuscript, the observed Tafel slope, is significantly influenced by the electrode ohmic resistance to support, which if not taken into consideration, will produce large fluctuations in the "Tafel slope" values reported in previous literature, summarized in Figure $3 \mathrm{c}$ in manuscript. 


\section{Reference}

(1) Lukowski, M. A.; Daniel, A. S.; Meng, F.; Forticaus, A.; Li, L.; Jin, S. Enhanced Hydrogen Evolution Catalysis from Chemically Exfoliated Metallic $\mathrm{MoS}_{2}$ Nanosheets. J. Am. Chem. Soc. 2013, 135, 10274-10277

(2) Xie, J.; Zhang, H.; Li, S.; Wang, R.; Sun, X.; Zhou, M.; Zhou, J.; Lou, X. W. D.; Xie, Y. Defect-Rich $\mathrm{MoS}_{2}$ Ultrathin Nanosheets with Additional Active Edge Sites for Enhanced Electrocatalytic Hydrogen Evolution. Adv. Mater. 2013, 25, 5807-5813

(3) Xie, J.; Zhang, J.; Li, S.; Grote, F.; Zhang, X.; Zhang, H.; Wang, R.; Lei, Y.; Pan, B.; Xie, Y. Controllable Disorder Engineering in Oxygen-Incorporated $\mathrm{MoS}_{2}$ Ultrathin Nanosheets for Efficient Hydrogen Evolution. J. Am. Chem. Soc. 2013, 135, 17881-17888

(4) Voiry, D.; Salehi, M.; Silva, R.; Fujita, T.; Chen, M.; Asefa, T.; Shenoy, V. B.; Dea, G.; Chhowalla, M. Conducting $\mathrm{MoS}_{2}$ Nanosheets as Catalysts for Hydrogen Evolution Reaction. Nano Lett. 2013, 13, 6222-6227

(5) Wang, H.; Lu, Z.; Xu, S.; Kong, D.; Cha, J. J.; Zheng, G.; Hsu, P.; Yan, K.; Bradshaw, D.; Prinz, F. B.; Cui, Y. Electrochemical tuning of vertically aligned $\mathrm{MoS}_{2}$ nanofilms and its application in improving hydrogen evolution reaction. PNAS. 2013, 110(49), 19701-19706

(6) Yu, Y.; Huang, S.; Li, Y.; Steinmann, S. N.; Yang, W.; Cao, L. Layer-Dependent Electrocatalysis of $\mathrm{MoS}_{2}$ for Hydrogen Evolution. Nano Lett. 2014, 14, 553-558

(7) Zheng, X.; Xu, J.; Yan, K.; Wang, H.; Wang, Z.; Yang, S. Space-Confined Growth of $\mathrm{MoS}_{2}$ Nanosheets within Graphite: The Layered Hybrid of $\mathrm{MoS}_{2}$ and Graphene as an Active Catalyst for Hydrogen Evolution Reaction. Chem. Mater. 2014, 26, 2344-2353

(8) Gopalakrishnan, D.; Damien, D.; Shaijumon, M. M. MoS 2 Quantum Dot-Interspersed Exfoliated $\mathrm{MoS}_{2}$ Nanosheets. ACS Nano. 2014, 8, 5297-5303

(9) Wang, H.; Lu, Z.; Kong, D.; Sun, J.; Hymel, T. M.; Cui, Y. Electrochemical Tuning of $\mathrm{MoS}_{2}$ Nanoparticles on Three-Dimensional Substrate for Efficient Hydrogen Evolution. ACS Nano. 2014, 8, 4940-4947

(10) Ma, C. B.; Qi, X.; Chen, B.; Bao, S.; Yin, Z.; Wu, X. J.; Luo, Z.; Wei, J.; Zhang, H. L.; Zhang, $\mathrm{H} . \mathrm{MoS}_{2}$ nanoflower-decorated reduced graphene oxide paper for high-performance hydrogen evolution reaction. Nanoscale. 2014, 6, 5624

(11) Ambrosi, A.; Sofer, Z.; Pumera, M. Lithium intercalation compound dramatically influences the electrochemical properties of exfoliated $\mathrm{MoS}_{2}$. Small. 2015, 11(5), 605-612

(12) Tao, L.; Duan, X.; Wang, C.; Duan, X.; Wang, S. Plasma-engineered $\mathrm{MoS}_{2}$ thin-film as an efficient electrocatalyst for hydrogen evolution reaction. Chem. Commun. 2015, 51, 74707473 
(13) Zhang, Z.; Li, W.; Yuen, M. F.; Ng, T.-W.; Tang, Y.; Lee, C.-S.; Chen, X.; Zhang, W., Hierarchical composite structure of few-layers $\mathrm{MoS}_{2}$ nanosheets supported by vertical graphene on carbon cloth for high-performance hydrogen evolution reaction. Nano Energy. 2015, 18, 196-204

(14) Deng, Z. H.; Li, L.; Ding, W.; Xiong, K.; Wei, Z. D., Synthesized ultrathin $\mathrm{MoS}_{2}$ nanosheets perpendicular to graphene for catalysis of hydrogen evolution reaction. Chem, Commun. 2015, 51, 1893-1896

(15) Yu, H.; Yu, X.; Chen, Y.; Zhang, S.; Gao, P.; Li, C., A strategy to synergistically increase the number of active edge sites and the conductivity of $\mathrm{MoS}_{2}$ nanosheets for hydrogen evolution. Nanoscale. 2015, 7, 8731-8738

(16) Li, H.; Tsai, C.; Koh, A. L.; Cai, L.; Contryman, A. W.; Fragapane, A. H.; Zhao, J.; Han, H. S.; Manoharan, H. C.; Abild-Pedersen, F.; Norskov, J. K.; Zheng, X. Activating and optimizing $\mathrm{MoS}_{2}$ basal planes for hydrogen evolution through the formation of strained sulphur vacancies. Nat. Mater. 2016, 15, 48-53

(17) Geng, X.; Sun, W.; Wu, W.; Chen, B.; Al-Hilo, A.; Benamara, M.; Zhu, H.; Watanabe, F.; Cui, J.; Chen, T. P., Pure and stable metallic phase molybdenum disulfide nanosheets for hydrogen evolution reaction. Nat.Commun. 2016, 7, 10672

(18) Cummins, D. R.; Martinez, U.; Sherehiy, A.; Kappera, R.; Martinez-Garcia, A.; Schulze, R. K.; Jasinski, J.; Zhang, J.; Gupta, R. K.; Lou, J.; Chhowalla, M.; Sumanasekera, G.; Mohite, A. D.; Sunkara, M. K.; Gupta, G. Efficient hydrogen evolution in transition metal dichalcogenides via a simple one-step hydrazine reaction. Nat. Commun, 2016, 7, 11857

(19) Ding, J.; Zhou, Y.; Li, Y.; Guo, S.; Huang, X. MoS 2 Nanosheet Assembling Superstructure with a Three-Dimensional Ion Accessible Site: A New Class of Bifunctional Materials for Batteries and Electrocatalysis. Chem. Mater. 2016, 28, 2074-2080

(20) Lin, L.; Miao, N.; Wen, Y.; Zhang, S.; Ghosez, P.; Sun, Z.; Allwood, D. A. SulfurDepleted Monolayered Molybdenum Disulfide Nanocrystals for Superelectrochemical Hydrogen Evolution Reaction. ACS Nano, 2016, 10, 8929-8937

(21) Tsai, C.; Li, H.; Park, S.; Park, J.; Han, H. S.; Norskov, J. K.; Zheng, X.; Abild-Pedersen, F. Electrochemical generation of sulfur vacancies in the basal plane of $\mathrm{MoS}_{2}$ for hydrogen evolution. Nat. Commun. 2017, 8, 15113

(22) Wu, X.; Wang, Z.; Yu, M.; Xiu, L.; Qiu, J. Stabilizing the MXenes by Carbon Nanoplating for Developing Hierarchical Nanohybrids with Efficient Lithium Storage and Hydrogen Evolution Capability. Adv. Mater. 2017, 29, 1607017

(23) Hu, J.; Huang, B.; Zhang, C.; Wang, Z.; An, Y.; Zhou, D.; Lin, H.; Leung, M. K. H.; Yang, S. Engineering stepped edge surface structures of $\mathrm{MoS}_{2}$ sheet stacks to accelerate the hydrogen evolution reaction. Energy Environ. Sci. 2017, 10, 593-603 
(24) Wang, D.; Zhang, X.; Bao, S.; Zhang, Z.; Fei, H.; Wu, Z. Phase engineering of a multiphasic $1 \mathrm{~T} / 2 \mathrm{H} \mathrm{MoS}$ catalyst for highly efficient hydrogen evolution. J. Mater. Chem. A. 2017, 5, 2681-2688

(25) Sun, Y.; Alimohammadi, F.; Zhang, D.; Guo, G. Enabling Colloidal Synthesis of EdgeOriented $\mathrm{MoS}_{2}$ with Expanded Interlayer Spacing for Enhanced HER Catalysis. Nano Lett. 2017, 17, 1963-1969

(26) Tang, C.; Zhong, L.; Zhang, B.; Wang, H. F.; Zhang, Q. 3D Mesoporous van der Waals Heterostructures for Trifunctional Energy Electrocatalysis. Adv. Mater. 2018, 30, 1705110

(27) Wang, Z.; Li, Q.; Xu, H.; Dahl-Petersen, C.; Yang, Q.; Cheng, D.; Cao, D.; Besenbacher, F.; Lauritsen, J. V.; Helveg, S.; Dong, M. Controllable etching of $\mathrm{MoS}_{2}$ basal planes for enhanced hydrogen evolution through the formation of active edge sites. Nano Energy. 2018, $49,634-643$

(28) Huang, L.-B.; Zhao, L.; Zhang, Y.; Chen, Y.-Y.; Zhang, Q.-H.; Luo, H.; Zhang, X.; Tang, T.; Gu, L.; Hu, J.-S. Self-Limited on-Site Conversion of $\mathrm{MoO}_{3}$ Nanodots into Vertically Aligned Ultrasmall Monolayer $\mathrm{MoS}_{2}$ for Efficient Hydrogen Evolution. Adv. Energy Mater. 2018, 8,1800734

(29) Xiong, P.; Ma, R.; Sakai, N.; Nurdiwijayanto, L.; Sasaki, T. Unilamellar Metallic $\mathrm{MoS}_{2} /$ Graphene Superlattice for Efficient Sodium Storage and Hydrogen Evolution. ACS Energy Lett. 2018, 3, 997-1005

(30) Wang, S.; Zhang, D.; Li, B.; Zhang, C.; Du, Z.; Yin, H.; Bi, X.; Yang, S. Ultrastable InPlane 1T-2H MoS 2 Heterostructures for Enhanced Hydrogen Evolution Reaction. Adv. Energy Mater. 2018, 8, 1801345 